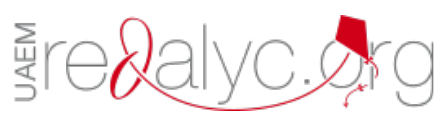

Centro Sur

ISSN: $2600-5743$

compasacademico@icloud.com

Grupo Compás

Ecuador

\title{
La educación digital en apoyo a la modalidad educativa presencial
}

Vera Mosquera, Jorge Francisco; Argüello Fiallos, Bélgica Elena; Obando Montenegro, José Enrique La educación digital en apoyo a la modalidad educativa presencial

Centro Sur, vol. 2, núm. 1, 2018

Grupo Compás, Ecuador

Disponible en: http://www.redalyc.org/articulo.oa?id=588861692005 


\title{
La educación digital en apoyo a la modalidad educativa presencial
}

\author{
Digital education in support of face-to-face education \\ Jorge Francisco Vera Mosquera jorge.veram@ug.edu.ec \\ Docente Universidad de Guayaquil, Ecuador \\ iD http://orcid.org/0000-0003-2934-0028 \\ Bélgica Elena Argüello Fiallos belgica.arguellof@ug.edu.ec \\ Docente Universidad de Guayaquil, Ecuador \\ (iD) http://orcid.org/0000-0001-5749-3609 \\ José Enrique Obando Montenegro jose.obandom@ug.edu.ec \\ Docente Universidad de Guayaquil, Ecuador \\ D http://orcid.org/0000-0002-0256-5916
}

Centro Sur, vol. 2, núm. 1, 2018

Grupo Compás, Ecuador

Recepción: 15 Marzo 2017 Aprobación: 31 Julio 2018

Redalyc: http://www.redalyc.org/ articulo.oa?id $=588861692005$
Resumen: La presente investigación se contextualizó en la Universidad de Guayaquil dentro del programa de investigación "reactivación del sistema de gestión de prevención de riesgos laborales", en su proyecto "socialización de la evaluación de riesgos, establecimiento de metas y plan de implementación”, del FCI (Fondo Competitivo de Investigación), avalizado por el Vice-Rectorado de Investigación, Gestión del Conocimiento y Postgrado en Guayaquil, Ecuador. La necesidad de capacitar en el área de seguridad y salud ocupacional en función de disponer de las certificaciones SETEC(Secretaría Técnica del Sistema Nacional de Cualificaciones Profesionales) al personal administrativo y de servicio de todo nivel en el ente universitario, cuyo número a la fecha oscila entre 5000 y 6000 personas, implica tener como objetivo un escenario educativo que facilite el proceso a llevar a cabo, por lo que la tecnología digital constituye un aliado educativo a la modalidad presencial o virtual que ha sido determinada por el personal responsable del proyecto, para el desarrollo de estos cursos. En el camino de la consecución de las conclusiones, se analizaron las concepciones tecnológicas actuales y su uso educativo, así como la necesidad de emplear la tecnología educativa, estimándose emprender el uso del gestor de aprendizaje Moodle, bajo el paradigma digital de aprendizaje en apoyo a las modalidades nombradas, dado que así se facilita el proceso educativo de cursos que se originan en sus distintas categorías y diversos destinatarios, dado el nivel de concurrencia cuyo número de accesos y consultas hace necesario prestar facilidades de aprendizaje al estudiante.

Palabras clave: Educación digital, tecnología educativa, modelo tradicional, gestor de aprendizaje.

Abstract: This research was contextualized in the University of Guayaquil within the research program "reactivation of the occupational risk prevention management system", in its project "socialization of risk assessment, establishment of goals and implementation plan", of the FCI (Competitive Research Fund), endorsed by the Vice-Rectorate for Research, Knowledge Management and Postgraduate Studies in Guayaquil, Ecuador. The need to train in the area of occupational safety and health in order to have the SETEC certifications (Technical Secretariat of the National System of Professional Qualifications) to the administrative and service staff of all levels in the university entity, whose number to date fluctuates between 5000 and 6000 people, it implies having as an objective an educational scenario that facilitates the process to be carried out, so that digital technology constitutes an educational ally to the face-toface or virtual modality that has been determined by the personnel responsible for the project, for the development of these courses. On the way to achieving the conclusions, the current technological conceptions and their educational use were analyzed, as well as 
the need to use educational technology, considering to undertake the use of the Moodle learning manager, under the digital paradigm of learning in support of the named modalities, given that this facilitates the educational process of courses that originate in their different categories and diverse recipients, given the level of competition whose number of accesses and consultations makes it necessary to provide learning facilities to the student.

Keywords: Digital education, educational technology, traditional model, learning manager.

\section{INTRODUCCIÓN}

La SETEC se creó el 17 de marzo del 2011, según el Artículo 7 del Decreto Ejecutivo $n^{\circ}$ 680, cuya publicación consta en el Registro Oficial No. 406 (OEI, 2012), definiéndose como: "una institución que trabaja arduamente por fortalecer el talento humano del país, mediante políticas públicas en el ámbito de la capacitación y certificación por competencias laborales, ejecutadas en todo el territorio nacional.”(SETEC, 2018)“.

La Universidad de Guayaquil, dentro de sus planes de mejoras consta el desarrollo e implementación de un sistema de gestión de prevención de riesgos laborales. Dicho trabajo está a cargo de la Dirección de Talento Humano a través de la Jefatura de Seguridad y Salud Ocupacional, de ahí surge la necesidad de llevar a cabo las capacitaciones necesarias del caso, a partir de septiembre del 2018, encontrándose al momento con un universo de 18 unidades académicas y 10 unidades administrativas, donde la población de las primeras oscila entre menos de 100 trabajadores y más de 1000, y de las segundas un número menor.

La población trabajadora total varía dependiendo de las necesidades institucionales, entre 5000 y 6000 personas, entre personal administrativo, docente y de servicio, lo que significa llevar a cabo un programa educativo continuo al respecto.

Indiscutiblemente el mundo vive la era digital, por lo que el ente universitario no se encuentra al margen de ello, (Comisión Europea, 2018) cita: "La educación y la formación son las mejores inversiones en el futuro de Europa. Desempeñan un papel primordial para impulsar el crecimiento, la innovación y la creación de empleo. ... La tecnología digital enriquece el aprendizaje de diferentes maneras y ofrece unas oportunidades de aprendizaje que deben estar al alcance de todos. Da acceso a un gran caudal de información y recursos. ".

La integración de toda capacitación con la tecnología digital debe manifestarse en las aulas y/o laboratorios que vayan a emplearse en las distintas facultades. La edad promedio del personal que hará de estudiantes es de 48 años, que indiscutiblemente se encuentran altamente influenciados por la enseñanza tradicional.

El aprendizaje electrónico (E-learning) surge en estas circunstancias y se conceptúa como (Segura-Robles \& Gallardo-Vigil, 2013): "completamente virtualizado, es decir, la instrucción se da a través de un dispositivo digital como un ordenador o dispositivo móvil ¿’”. Los mismos autores mencionan como características básicas del mismo lo siguiente: 
- Interactividad: Quien usa la plataforma debe estar consciente que es uno mismo el responsable de su aprendizaje.

- Flexibilidad: El sistema debe adaptarse a las distintas circunstancias estructurales físicas y educativas.

- Escalabilidad: Se puede trabajar con distinto número de usuarios.

- Estandarización: El sistema permita utilizarse y/o actualizarse por terceros para replicar.

Por lo que un programa de capacitaciones al disponer de estas características, simplemente se adapta a los cursos y usuarios, independiente de su número.

\section{MATERIALES Y MÉTODOS}

Vale estimar, la presencia y uso de la internet en el centro educativo, que ha creado el inicio de un cambio, que se ve influenciado por un aprendizaje en la red, que se conceptúa como E-learning empresarial, (Esteban-Albert \& Zapata-Ros, 2016) que los autores citan: "Se trata de lo que comúnmente se conoce como el e-learning empresarial o del sector empresarial, que pone en circulación materiales de aprendizaje más o menos asistidos por un auténtico sistema de aprendizaje en redes. En realidad es esta la auténtica esencia del E-learning para muchos, donde se distingue la distribución de contenidos más o menos asistidos (se entiende asistidos de forma automática) y el sentido instrumental de éstos en un contexto educativo." (pág. 3).

Esto hace que el quehacer pedagógico se tenga que renovar para que tenga efecto la integración curricular de la tecnología, surgiendo así el paradigma de la educación digital (Telefónica, 2015): "Los expertos coinciden en afirmar que el modelo educativo actual está desfasado para la era digital. El fracaso escolar o el desajuste con el mercado laboral parecen corroborarlo. Se impone por tanto, un nuevo paradigma" (pág. 4).

Vale considerar la visión que regularmente se tiene de la tecnología a nivel general docente (Noëlle \& Granados, 2015): "El hecho de que las TIC respondan casi exclusivamente a unos criterios de utilidad y de eficacia que no tienen nada que ver con los sistemas económicos, políticos y/o culturales de nuestra sociedad, evidencia un discurso tecnocentrista por parte de los estudiantes" (pág. 16), por lo que la didáctica a emplearse debe partir de un enfoque innovador de formación pedagógica/ andragógica, que trate un planteamiento didáctico de cómo utilizar la tecnología en clase.

\section{RESULTADOS}

Lo mencionado, implica crear ambientes de aprendizaje (Diaz-Barriga, 2013) , a lo que el autor cita: "Un ambiente de aprendizaje es el resultado de establecer secuencias didácticas que ofrecen un ordenamiento de acciones a realizar, no necesariamente en forma única” (pág. 7). 
Esto implica reconocer los Ambientes personales de aprendizaje (PLE - Personal Learning Environment): (Adell \& Castañeda, 2010) cuyo concepto los autores citan así: "conjunto de herramientas, fuentes de información, conexiones y actividades que cada persona utiliza de forma asidua para aprender" (pág. 7). A su vez orientan sus elementos en:

\# Dónde acceder a la información:

o Sitios de publicación (blogs, wikis), repositorios y bases de datos de audio (iTunes U, podcasts), vídeo (YouTube, Vimeo, Blip, etc.), multimedia (Slideshare, repositorios digitales como el de National Geographic, BBC u otras empresas de divulgación), objetos de aprendizaje estandarizados (AGREGA, MERLOT, repositorios de diferentes instituciones formales), lectores de RSS (Google Reader, RSSowl, etc.), sitios de noticias, portales de información específica, repositorios OpenCourseWare, etc.

\# Dónde modificar la información:

o Wikis, suites ofimáticas de escritorio (OpenOffice.org) y en red (GDocs, Zoho), herramientas de mapas mentales (CMapTools, Creatively, Collaborilla), herramientas de edición de audio, de vídeo, creación de presentaciones, mapas conceptuales, cronogramas y en general cualquier tipo de artefacto informacional.

\# Dónde relacionarme con otros; lo constituye la red personal de aprendizaje en red (Personal Learning Environment - PLN).

En esta última red, es que se ubica el gestor de aprendizaje.

Los autores del presente trabajo de investigación recomiendan el empleo de Moodle, por su utilidad para el aprendizaje: (Lechuga \& Rojas, 2016) "Al utilizar la plataforma Moodle como herramienta para el aprendizaje constructivista, los educandos tienen la oportunidad de ampliar su experiencia; esta tecnología le ofrece opciones para lograr que el aula tradicional se convierta en un nuevo espacio, en donde tienen a su disposición actividades innovadoras de carácter colaborativo y con aspectos creativos que les permiten afianzar lo que aprenden, además de construir su conocimiento en conjunto con el docente en su rol de facilitador, otorgándole la libertad necesaria para que explore el ambiente tecnológico, pero estando presente cuando tenga dudas o le surja algún problema, siempre enfatizando para hacerlos pensar, reflexionar, investigar, estudiar y comprender." (pág. 16).

Por ende, se debe considerar el diseño de material para puesta en línea cuya aplicación obedezca al contexto y a un diseño instruccional del proceso enseñanza-aprendizaje a emplearse. Así, se tiene que (Romero, Sola, \& Trujillo, 2015): "Desde el punto de vista de la intención didáctica, se pueden diseñar materiales para exposición de contenidos, para plantear actividades de enseñanza-aprendizaje, o bien para integrar en un mismo objeto educativo ambas cuestiones dando lugar a lo que denominamos materiales integrados" (pág. 63), debiendo respetarse siempre la autoría de los mismos. El empleo de un modelo híbrido potencia el trabajo del tutor, es decir, el uso de la virtualidad en apoyo a la modalidad presencial, hace que el facilitador oriente su quehacer educativo, en base a las metodologías de aprendizaje identificadas en el diagnóstico de estilos 
de los estudiantes, que deben ser realizados a los aprendices al inicio de los procesos educativos en el primer curso que tomen.

\section{DISCUSIÓN}

Al disponerse de los cursos debidamente categorizados por los distintos aspectos a tratarse dentro del programa de capacitaciones a dictarse, vale que el modelo pedagógico a implementarse, considere el paradigma digital. Los contenidos deben ser desarrollados e implementados dentro de una enseñanza programática que contemple los ambientes personales de aprendizaje personales y en red que los estudiantes del personal del centro educativo dispongan y puedan construir para su aprendizaje autónomo y colaborativo. El empleo del gestor de aprendizaje Moodle, no solo garantizará el proceso digital sino también el educativo, dado que emplea paradigmas como el constructivismo social, aprendizaje significativo y los aprendizajes autónomos y colaborativos. Todo estudiante iniciante debería de ser evaluado previo a la toma de los cursos que tome, dado que así el facilitador que le corresponda en cualquiera de los mismos, tiene un panorama colectivo e individual de sus estudiantes. Igualmente, al inicio, es recomendable realizar una encuesta digital para que los facilitadores puedan identificar, reconocer y/o armar los ambientes de aprendizaje.

Los actos didácticos que los tutores empleen en modalidad presencial, estarán respaldados con los contenidos de curso que se dispongan en el gestor de aprendizaje, por lo que sus actividades las puede pulsar como recrear en el momento que lo considere necesario didácticamente. La evaluación está a potestad de la persona tutora en el proceso, pudiendo apoyarse en el gestor de aprendizaje Moodle, con sus distintas formas de evaluar, en la forma e instancias que desee: antes, durante y al final del proceso educativo.

\section{Referencias}

Adell, J., \& Castañeda, L. (2010). Los Entornos Personales de Aprendizaje(PLEs): una nueva manera de entender el aprendizaje. En Roig Vila, R. \& Fiorucci, M. (Eds.). Claves para la investigación en innovación y calidad educativas. La integración de las Tecnologias de la informacion y la comunicación y la interculturalidad en las aulas. Alcoy, España: Marfil

Comisión Europea. (2018). Comunicación De La Comisión Al Parlamento Europeo, Al Consejo, Al Comité Económico Y Social Europeo Y Al Comité De Las Regiones sobre el Plan de Educación Digital. Recuperado de https://eur-lex.europa.eu/legal-content/ES/ TXT/?uri=COM\%3A2018\%3A22\%3AFIN

Diaz-Barriga, Á. (2013). TIC en el trabajo del aula. Impacto en la planeación didáctica. RIES - Revista Iberoamericana de Educación Superior, 10(4), 3-21. Recuperado de http://www.redalyc.org:9081/ articulo.oa?id=299128588003 
EcuRed. (2018). Informatica Educativa. Cuba: EcuRed. Recuperado de https:// www.ecured.cu/Inform\%C3\%A1tica_Educativa

Esteban-Albert, M., \& Zapata-Ros, M. (2016). Estrategias de aprendizaje y eLearning. Un apunte para la fundamentación del diseño educativo en los entornos virtuales de aprendizaje. RED. Revista de Educación a Distancia.50(1), 1-12. doi:http://dx.doi.org/10.6018/red/50/15

La redacción. (1 de septiembre de 2013). Las TIC van a la escuela. España: Investigación $\mathrm{y}$ Ciencia. Recuperado de https://www.investigacionyciencia.es/revistas/ investigacion-y-ciencia/el-amanecer-de-los-exoplanetas-582/las-tic-vana-la-escuela-11358

Lechuga, A., \& Rojas, A. (2016). MOODLE COMO HERRAMIENTA DE COMUNICACIÓN Y ENSEÑANZA APRENDIZAJE, DESDE UN ENFOQUE CONSTRUCTIVISTA. $R D U$ - Revista Digital Universitaria.17(11),1-18. Recuperado de http://www.ru.tic.unam.mx/bitstream/handle/123456789/2687/ art79_2016.pdf?sequence $=1$ \&isAllowed $=y$

López, C., Benedito, V., \& León, M. (2016). El Enfoque de Competencias en la Formación Universitaria y. Formación Universitaria, 9(4), 11-22. doi: $10.4067 /$ S0718-50062016000400003

Noëlle, M., \& Granados, J. (2015). Orientaciones para la formación didáctica del profesorado novel en las TIC: los entornos 2.0 y el trabajo colaborativo en el aula. Referencia Pedagógica, 3(1), 13-26. Recuperado de http:// rrp.cujae.edu.cu/index.php/rrp/article/view/77/91

OEI. (2012). EUROSOCIAL PROGRAMA PARA LA COHESIÓN SOCIAL EN AMÉRICA LATINA. Recuperado de http://sia.eurosocial-ii.eu/ files/docs/1412243797-Informe\%20final\%20SNCP\%20Ecuador.pdf

Reyes, F., Vera, L., \& Colina, E. (2014). Estrategias creativas para promover el aprendizaje significativo en la práctica docente simulada. Opción,75(1), 55-74. Obtenido de http://www.redalyc.org/pdf/310/31035400002.pdf

Riesco, M., Fondón, M., Álvarez, D., López, B., Cernuda, A., \& Aquilino, J. (2018). Informática: materia esencial en la educación obligatoria del siglo XXI. ReVisión. 7(3). Obtenido de http://www.aenui.net/ojs/index.php? journal $=$ revision\&page $=$ article\&op $=$ viewArticle\&path\%5B $\% 5 \mathrm{D}=162 \&$ path\%5B $\% 5 \mathrm{D}=270$

Romero, J., Sola, T., \& Trujillo, J. (2015). Posibilidades didácticas de las herramientas Moodle para producción de cursos y materiales educativos. Digital EDUCATION,28(1),59-76. Recuperado de http:// revistes.ub.edu/index.php/der/article/view/14092/pdf

Segura-Robles , A., \& Gallardo-Vigil, M. (2013). ENTORNOS VIRTUALES DE APRENDIZAJE: NUEVOS RETOS EDUCATIVOS. Revista cientifica electrónica de Educación y Comunicación en la Sociedad del Conocimiento, 12(2), 260-272. Recuperado de http://www.eticanet.org/ revista/index.php/eticanet/article/view/34/30

SETEC. (2018). Qué es la SETEC. Recuperado de http:// www.cualificaciones.gob.ec/que-es-la-setec/

Sharples, M. (Abril de 2018). Pedagogía en línea. Un análisis del viaje de la educación hacia el entorno digital. Educación Digital. 540(1), 90-95. Recuperado de https://www.investigacionyciencia.es/revistas/especial/ educacin-digital-734/pedagoga-en-lnea-15322 
Soto, J., \& Torres, C. (2016). Percepciones y expectativas del aprendizaje en jóvenes universitarios. REDU - Revista de Docencia Universitaria.14(1),51-67. Recuperado de https://polipapers.upv.es/ index.php/REDU/article/view/5797/6350

Telefónica. (Abril de 2015). El nuevo paradigma de la educación digital.A un click de las TIC. Recuperado de https://aunclicdelastic.blogthinkbig.com/wp-content/uploads/Elnuevo-paradigma-de-la-educaci\%C3\%B3n-digital.pdf

Vera, P., Baquedano, C., Ferrám, Y., Olavarría, S., Parra, E., \& De Souza, B. (2008). Una innovación pedagógica para la formación de universitarios emprendedores. Revista da FAE,11(2), 113-126. Obtenido de https:// revistafae.fae.edu/revistafae/article/view/331/227

Viera, J., Hernández, J., Castillo, J., \& Domínguez, R. (18 de Noviembre de 2016). Las tecnologías móviles en ámbito universitario . En III Jornadas Iberoamericanas de Innovación Educativa en el ámbito de las TIC .Conferencia llevado a cabo en Las palmas de Gran Canaria. Recuperado de https://acceda.ulpgc.es/ bitstream/10553/20447/1/0730076_00000_0001.pdf 